\title{
Novel wearable VCSEL-based blood perfusion sensor
}

\author{
E. Zherebtsov ${ }^{1}$, S. Sokolovski ${ }^{1}$, V. Sidorov ${ }^{2}$, I. Rafailov ${ }^{4}$, A. Dunaev ${ }^{3}$, E.U. Rafailov ${ }^{1}$ \\ ${ }^{1}$ Aston Institute of photonic technologies, Aston University, Birmingham, UK \\ ${ }^{2}$ SPE “LAZMA” Ltd., Moscow, Russia, 125252 \\ ${ }^{3}$ Research and Development Center of Biomedical Photonics, Orel State University, Orel, Russia \\ ${ }^{4}$ Aston Medical Technology Ltd., Birmingham, UK \\ e-mail: e.zherebtsov@aston.ac.uk
}

\begin{abstract}
A wearable $850 \mathrm{~nm}$ VCSEL-based blood perfusion sensor operating on the principles of laser Doppler flowmetry (LDF) and Dynamic Light Scattering (DLS) has been developed and tested. The sensitivity of the sensor to changes in skin blood perfusion has been demonstrated.
\end{abstract}

Keywords-Laser Doppler flowmetry, dynamic light scattering, skin blood perfusion, haemodynamic, capillaries.

\section{INTRODUCTION}

The wearable implementation of LDF can become a truly new diagnostic interface to monitor cardiovascular parameters, which could be of interest for prediction of CVD [1, 2], as well as in sports and rehabilitation medicine. A fine analysis of capillary blood flow structure and rhythm in the time and frequency domains, coupled with a new possibility of round-theclock monitoring, can provide valuable diagnostic information about stressful conditions in the body, sleep quality, the effects of smoking and endothelial dysfunction, which is usually a herald of more severe CVD [3-5]. In this study, we characterise an in-house built sensor in a series of experiments on healthy volunteers.

\section{EXPERIMENT AND RESULTS}

The developed experimental prototype of the wearable sensor uses VCSEL die chips $(850 \mathrm{~nm}, 1.4 \mathrm{~mW} / 3.5 \mathrm{~mA})$ as a single mode laser source. Fibre-free solution and direct illumination of tissue by the laser diode make it possible to avoid fibre coupling losses as well as to decrease the movement artefacts which are common in fibre-based LDF monitors. To find a correlation between the changes in the registered blood perfusion and actual body movements the integral accelerometer has been embedded in the sensor.

To evaluate the sensitivity of the sensor to changes in skin blood microperfusion, a series of experiments was conducted in healthy volunteers. During this study, the sensor was placed on the dorsal wrist. In the first step, the patterns of skin blood perfusion in the wrist were registered in rest. Signal processing based on 1-D wavelet transform has shown that the proposed sensor can detect heartbeat rate by analysis of the small arteries blood flow rhythms in the frequency domain. A conducted series of breath holding tests have shown that the average amplitude of the response incisura in the signal achieves $25 \%$ of the initial level, which is comparable with the results of the conventional LDF monitors. In the second step, the response on the occlusion test has been investigated. Occlusion tests were performed according to standard procedures: the volunteer was in a sitting position (forearm, at heart level), the cuff of the tonometer was located on the upper arm near the area of the shoulder and the cuff was pumped to a pressure of $220-230 \mathrm{mmHg}$. The conducted test has confirmed the ability of the sensor to register skin blood perfusion in the physiological range with a demonstration of physiological zero during the arm cuff occlusion. An implemented filtering technique taking into account the accelerometer data allowed the wearable sensor system to significantly increase the signal to noise ratio value and decrease the movement artefacts to an acceptable level. The collection of the advantages present in the LDF system introduced into a wearable format demonstrates that this technology can completely replace the pulse sensors based on light reflection currently present in all modern fitness bracelets and smart watches.

\section{ACKNOWLEDGMENT}

This publication is part of a project that has received funding from the European Union's Horizon 2020 research and innovation programme under the Marie Skłodowska-Curie grant agreement No 703145 .

\section{REFERENCES}

[1] A. Goltsov et al., "Bifurcation in blood oscillatory rhythms for patients with ischemic stroke: A small scale clinical trial using laser Doppler flowmetry and computational modeling of vasomotion," Front. Physiol. 8(MAR), 1-11 (2017).

[2] K. Farkas et al., "Non-invasive assessment of microvascular endothelial function by laser doppler flowmetry in patients with essential hypertension," Atherosclerosis 173(1), 97-102 (2004).

[3] A. Dunaev et al., "Investigating tissue respiration and skin microhaemocirculation under adaptive changes and the synchronization of blood flow and oxygen saturation rhythms," Physiol. Meas. 35(4), 607621 (2014).

[4] T. Takahashi, Y. Takikawa, and R. Kawagoe, "Differences in the Pulsatile component of the skin hemodynamic response to verbal fluency tasks in the forehead and the fingertip," Sci. Rep. 6 (Feb.), 1-7, Nature Publishing Group (2016)

[5] V. Dremin et al., "Laser Doppler flowmetry in blood and lymph monitoring, technical aspects and analysis," in Progress in Biomedical Optics and Imaging - Proceedings of SPIE 10063 (2017). 